\title{
Urban Wetland Restoration Design Based on the Ecological Concepts--Taking Guanyintang Wetland Park in Chongqing as an Example
}

\author{
Qing Zhang ${ }^{l}$, Weiran Tian ${ }^{l}$, Ying Huang ${ }^{l, *}$ \\ ${ }^{1}$ School of Tourism and Landscape Architecture, Guilin University of Technology, 541006 Guilin, Guangxi, China
}

\begin{abstract}
With the improvement of people's awareness of environmental protection and the pursuit of high standards of living places, there are more thinkings about urban ecological issues. Through the construction of urban wetland parks, it is of great significance to improve the appearance of the city and maintain the urban ecological balance and sustainable ecological development. Taking Guanyintang Wetland Park in Chongqing as an example, low-lying swamps are used to build wetland parks. Guided by ecological theory, make full use of the original site conditions to restore the design of the wetland park, optimize the ecological functions of the area, and increase the recreation of tourists places, elaborately create an urban environment with perfect functions, beautiful ecology, livable and industrial, so as to further enhance the city's taste.
\end{abstract}

\section{Introduction}

The urban wetland park is the ecological system with the greatest urban biological advantage, which plays a key role in urban ecological protection, environmental improvement, and climate regulation. And the sustainable development of urban ecology is guided by the rich and diverse species ${ }^{[1]}$. With the rapid population growth, the scale of the city has expanded significantly, but the wetlands around the city and its surroundings have gradually shrunk, the ecosystem has been destroyed, and the urban environment has been severely tested. It is also getting more and more urgent to protect the ecological environment, restore urban wetlands, and build wetland parks. Therefore, the design of urban wetland park based on the ecological concept has important practical significance for wetland restoration, landscape design and organization of recreational sites.

\section{Principles of urban wetland park design}

\subsection{Ecological principles}

Wetlands have the basic ecological functions of regulating water cycle,improving water quality, protecting biodiversity, and maintaining sustainable ecological development, which can improve the long-term urban environment,water resources destruction, and biological resource reduction if it is combined with urban construction and restoration of wetland parks. The urban wetland park not only protects and stabilizes the ecological environment of the wetland area, but also plays a significant role in ecological compensation and balance of the surrounding environment.Therefore, the implementation of ecological principles can realize the sustainable development of wetland parks, and improve the ecological benefits of the entire city.

\subsection{Scientific design principles}

Choosing suitable sites and planting suitable plants are an important part of the planning and design of urban wetland parks. Choosing a suitable site to construct an urban wetland park plays a key role in the balance of urban ecology and the protection of wetland resources. In the planning and design of wetland parks, master the geomorphological and hydrological characteristics of the area where the wetland is located, adapt measures to local conditions, adapt the trees to the site, and select native tree species to highlight the regional characteristics ${ }^{[2]}$.

In order to effectively combine natural ecosystems with artificial interventions, minimize environmental damage and impact, protect and optimize wetland ecosystems, scientific and reasonable design is required to coordinate natural ecosystems and artificial planning environments ${ }^{[3]}$. According to the site characteristics, the landscape design of the wetland park and its basic ecological functions can be adapted and coordinated to achieve the goal of simultaneous development of ecology and landscape to meet the requirements of wetland ecological protection, entertainment and leisure, and popular science education.

\footnotetext{
*Corresponding author: 490498081@qq.com
} 


\subsection{Aesthetic principles}

Urban wetland park should combine artistic and cultural features. For example, the arrangement of plants should conform to the aesthetic principle of rhythm, contrast and balance, and transitional response; the design of architectural sketches should have aesthetic ideas as well as the historical and cultural connotation of the city, reflecting the cultural landscape of the wetland park . The cultural landscape of the urban wetland park is the restoration and cultural reproduction of the city's historical features, which makes the urban wetland park a new business card that symbolizes the unique historical landscape features and cultural connotations of the city.

\section{Case study-design of Guanyintang Wetland Park in Chongqing}

\subsection{Background of wetland restoration}

The original terrain of the Guanyintang Wetland is mostly hilly and low-lying swamps. A secondary wetland landscape is formed after the natural rise of the river. The natural vegetation is mainly deciduous broad-leaved forests, followed by bamboo forests, where many animals inhabit here, which has the potential to build a national-level urban wetland park. However, due to the destruction of the wetland caused by urban construction and industrial development, the good wetland environment has gradually degraded into grassland and shrubs, and the farmland has been overgrown with weeds, causing serious water pollution. In response to the call for the construction of urban ecological civilization and the proposed construction goal of the "Chongqing Green Island New District", the Guanyintang Wetland has been protected and developed, the park has been renovated and the wetland ecology has been restored.

\subsection{Overview of the study area}

Bishan District, Chongqing City is known as the "West Gate of Chongqing", has a history of more than 1,200 years. The Guanyintang Wetland Park is located in the southern part of Bishan District, Chongqing City. The specific location is Guanyintang Village, close to the Binan River. The geographical coordinates are $106^{\circ} 22^{\prime} \mathrm{E}$ and $29^{\circ} 55^{\prime} \mathrm{N}$. The total area of the wetland park is $40.53 \mathrm{hm}^{2}$.

\subsubsection{Topography}

Bishan District is located in the upper reaches of the Yangtze River in Chongqing. The geomorphology is divided into a humid mid-subtropical zone and is located in a parallel low mountain valley in the east of Sichuan. The topography of Guanyintang Village in Bishan District selected by the Wetland Park is mostly hilly and low-lying swamps.

\subsubsection{Climate characteristics}

The area of Guanyintang Wetland Park is affected by the humid mid-subtropical monsoon climate throughout the year, with humid climate, abundant rainfall, four distinct seasons, long frost-free period, high humidity, and many cloudy and rainy characteristics. The annual average temperature is $18^{\circ} \mathrm{C}$, the average frost-free period is 338 days, and the average relative humidity is about $81 \%$. The average rainfall is $1064.7 \mathrm{~mm}$. The distribution of rainfall is uneven during the whole year. And the main rainfall period is from May to August each year.

\subsubsection{Hydrological resources}

The total water area of the park is about $13.94 \mathrm{hm}^{2}$, accounting for one third of the whole area of Guanyintang Wetland Park. The tidal flat wetland area is $1.84 \mathrm{hm}^{2}$, the river wetland area is about $4.25 \mathrm{hm}^{2}$, and the central water area is about $7.85 \mathrm{hm}^{2}$, with the deepest water reaching $7 \mathrm{~m}$. The wetland resources in the park are composed of original ecological wetlands (Fig.1) and artificial wetlands. The wetland types include the original Binan River, Shuangcha River, farmland and mudflats, artificial trenches, Guanyintang and Lianhua Reservoir. Wetland resources are classified into: 2 first-level categories, 3 second-level categories, and 5 third-level categories ${ }^{[4-5]}$. The water resources are integrated by artificial means to form a complete water area of the park (Fig.2). The control area of the river in the park is mainly the Binan River. The length of the control section of the river is about $635 \mathrm{~m}$. The control of the drainage area of the river broadens the ecological control range of the wetland.

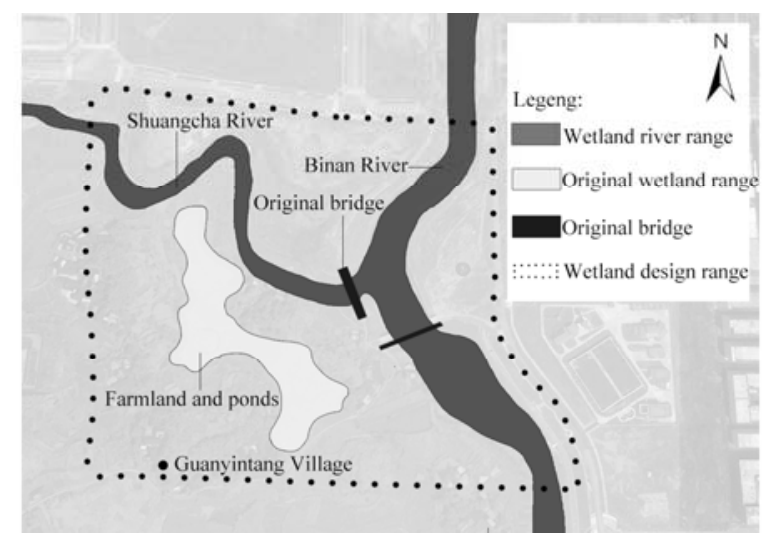

Fig. 1. Original Wetland Water Area (before design). 


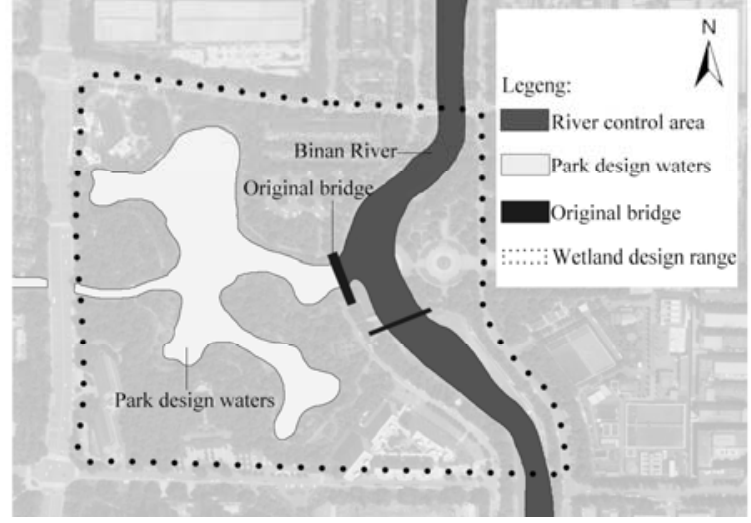

Fig. 2. Overall Water System of the Park (after design).

\subsection{Design and solve problems}

Guided by ecological theory, the transformation design of Guanyintang Wetland Park will be carried out to improve and restore the wetland ecology. Hilly lands and low-lying swamp lands are both challenges and opportunities in the design. Making full use of site conditions to plan routes and design site functions is the core to design the wetland park. According to the site conditions and the design principles of the urban wetland park, the key issues to be solved in the analysis of the park are as follows:

- How to protect the biological habitat?

- How to organize the plant landscape based on the existing vegetation?

- How to inherit and Continue urban culture and history?

- How to make the venue serve people?

These problems provide a realistic basis for the realization of the ecological restoration and transformation design of Guanyintang wetland.

\subsection{Wetland park ecological restoration design}

The design of Guanyintang Wetland Park takes the Millennium Bayu culture as the background, incorporates the design concept of "nature, culture and the future", and adheres to the sustainable ecological principle of "people-oriented, harmony between man and nature", integrating ecology, art and culture into one, it reproduces the "Eight Views of Biyi" in the long history and culture of the Millennium city ${ }^{[6]}$, and coordinates the wetland restoration goals, site characteristics and wetland landscape design to create a "spiritual home" of poetic habitat and display the profound historical and cultural heritage of the Bishan area ${ }^{[7]}$. In order to solve the practical problems of Guanyintang Wetland Park, the design and division of functional areas(Fig.3) include: wetland dense forest protection area, wetland water scenic area,cultural landscape area,popular science education area, leisure and entertainment area .

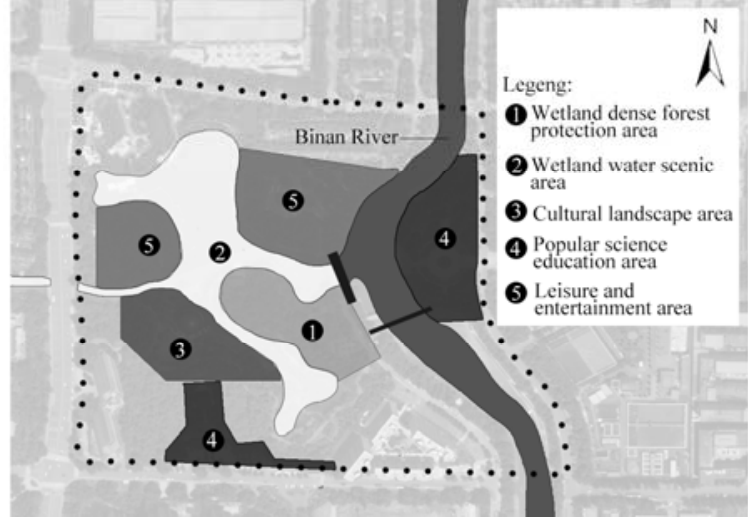

Fig. 3. Division of Functional Areas.

\subsubsection{Wetland dense forest protection area}

The wetland dense forest reserve is at the center of the wetland, facing water on three sides and dense vegetation, forming a semi-enclosed and quiet environment. It is a core habitat for rare animals and plants and a key wetland protection area. In the road design, forest footpaths and forest viewing platforms are specially set up to organize the flow of people to watch the birds and the scenery to reduce the impact of crowd activities on the reproduction and habitat of animals and plants. The native plant community in the dense forest reserve has a prominent landscape. The design gives priority to retaining the original plants and adding a plant transition zone to interweave the natural wildness and exquisite details so as to highlight the community combination mode of "learning from nature". There is a good composition of plants in the reserve and other regions. Its wetland environment formed has attracted more than 30 species of wild birds to inhabit here.

In addition, rare plants, endemic plants, and rare and endangered plants are also planted in the reserve, Such as Manglietia insignis, Michelia wilsonii, Osmanthus fragrans, Ottelia acuminata, etc. ${ }^{[8]}$. The overall design of the plant landscape in the park uses aesthetic principles ingeniously to combine the landscape space with the artistic conception. The close-range, middle-range, long-range and arbor, shrub, and grass layers of plants are arranged with blank spaces, and the community structure is clear. Planting evergreen, deciduous or flower-viewing, leaf-viewing and fruit-viewing plants during the four seasons makes spring, summer, autumn and winter different seasons.

\subsubsection{Wetland water scenic area}

There is a large elevation difference between the hilly land and the swamp land. The height difference is used to design the overlapping waterfall and the traditional water management method is continued to make the water in the park form a flowing natural circulating water system, so that the park water environment system has the ability of self-repair and self-renewal ${ }^{[9]}$, not only ensured the water quality of the wetland, but also played a role in flood regulation and storage during the flooding period. The wetland park waters, revetments, waterfront 
roads and tidal flats are all natural design forms, implementing the design concept of the park.

Wetland water scenic spots have various types of landscapes, such as a large area of microwave rippling water reflecting lush waterfront plants, and shallow waters where the water is crystal clear and you can watch underwater plants and fish and shrimp play. The waterfall has a $30 \mathrm{~m}$ drop, and its majestic momentum adds agility to the park. It is supplemented by stone ladders that can climb up to the mountain pavilion. "Five-stacked Spring" imitates the natural stacked spring, creating an artistic conception of "the cicadas noisy forests are more quiet, then the birdsong mountain is more secluded". The surrounding plants are diverse and scattered. The flow and sound of the stacked water vary with the seasons and rainfall. The water music fountain landscape covers an area of $2.1 \mathrm{hm}^{2}$, and the water column of the fountain can reach up to $68 \mathrm{~m}$. The perfect combination of water, light and color gives the wetland park a taste.

\subsubsection{Cultural landscape area}

Cultural landscape design can fully inherit and continue urban culture and history, which is the highlight of urban wetland park design. The cultural landscape area of Guanyintang Wetland Park is mainly about the Bayu civilization, and displays historical stories, cultural celebrities, poetry and paintings, etc. relying on antique buildings or sculptures. For example, the built "Donglin Pavilion", "Hufeng Tower", "Maolaixuan", and Umbrella Pavilion are named after "Biyi Eight Scenic Spots", symbolizing the reconstruction of historical sites; the famous scenic spot "Number One Scholar Bridge" in the park is carved with beams and painted buildings, with raised cornices and corners, and there are engraved with famous poems, calligraphy and paintings on both sides of the internal beams, showing the thousand-year ancient city culture. The pavilions and pavilions in the park have an antique building area of about $5,200 \mathrm{~m}^{2}$, all of which are in the form of eastern Sichuan wood structure to reproduce the architectural style of Bayu during the Ming and Qing Dynasties.

\subsubsection{Popular science education area}

The Popular science education area provides citizens with a base to popularize wetland culture and promote ecological protection. In this area, an ecological science museum and a wetland culture exhibition hall are built here, sketches inspired by ecological and environmental protection, and feature wetland plants can be configured. For example, the ecological and landscape coordination in Guanyintang Wetland Park, the Aquatic Ecology Science Museum, Dinosaur Fossil Wall, Turtle Shell Sculpture and many other sculptures convey cultural and scientific and educational significance. The Science Museum also has a decorative function. Its appearance is hemispherical, and the wall is a sea-blue glass curtain wall. It is located on the edge of the wetland and is unique among the many antique buildings in the park.
Featured aquatic plants are planted around the Science Museum, such as Cyperus papyrus and Thalia dealbata Fraser, which are introduced from abroad.

\subsubsection{Leisure and entertainment area}

The leisure and entertainment area is widely distributed, including a variety of landscapes, such as: leisure square landscape, waterfront recreation landscape, plant landscape, and sketch landscape. Each landscape should be in line with the core design concept of the wetland park, integrate ecology, art, and culture, and be designed to serve people. For example, the recreational area of the park provides recreational facilities and venues while designing plants.It protects the original FicusvirensAit.var.sublanceolata in the wetland, and uses low shrubs and stools to enclose the design as a solitary tree. Consider ancient tree protection and service facilities. The arrangement of entertainment facilities and leisure seats in the leisure square is based on the premise of people's needs, enhancing the interaction between people and the wetland landscape, allowing people to get close to nature, feel the wetland style in the city, and relax and feel happy in the park.

\section{Conclusions}

From the restoration design of Guanyintang Wetland Park, it can be seen that it has significant effect in protecting biodiversity, degrading pollution, purifying the air, and alleviating the urban "heat island effect", which has significantly improved the regional ecology. Urban wetland park is an important model to improve the urban ecological environment. However, due to different urban environment and conditions, and the construction of urban wetland parks are still facing many challenges. Based on the previous exploration and research, the innovative design method is the research focus of wetland restoration design based on the ecological basis of urban wetland parks in the future, . Guanyintang Wetland Park is a model of urban wetland parks in Southwest China. The example of Bayu cultural landscape in the park is a successful case of urban wetland park ecological concept combined with cultural design. It is hoped that the wetland restoration design can provide a useful reference for the design of Urban Wetland Park.

\section{References}

1. H.Wang, Landscape planning and design. $2^{\text {nd }}$ edition, Southeast University Press, (2009).

2. X.H.Su, Plant landscape planning and design, China Forestry Publishing House, (2012).

3. J.Meng, Discussion on urban wetland park planning under the concept of ecological planning, Theoretical Research on Urban Construction (Electronic Edition), no.01, 40-41, (2017). 
4. B.Huang, X.M.Liu,S.X.Huang, et al, Research on the Evaluation System of Guanyintang Urban Wetland Park in Bishan County, Landscape Architecture, no.06, 140-143, (2013).

5. T.R.He, The problems and countermeasures in the utilization of wetland resources in Chongqing, Journal of Chongqing Normal University (Natural Science Edition), no.02, 60-63, (2002).

6. X.C.Guo, L.R.Zeng, Viewing the Concept and Principles of Ecological Design from the Landscape Design of Urban Wetland Parks: Taking Chongqing Bishan Guanyintang Wetland Park as an example, Ecology and Economics, 30(05), 196-199, (2014).

7. L.Cai, Guanyintang wetland park integrating "ecology + culture + ancient architecture", April-2020. http://www.chinavalue.net/Finance/Blog/2020-4-18/ 1857939.aspx.

8. B.Huang, T.Chen, X.M.Liu, et al, Biodiversity Evaluation and Protection Countermeasures of Guanyintang Urban Wetland Park in Bishan County , Journal of Hunan Agricultural University(Natural Science Edition), 40(01), 37-42, (2014).

9. Y.S.Xie, Considerations on the construction of urban ecological wetland parks based on the construction of ecological civilization, Southern Agricultural Machinery, 50(02), 240, (2019). 